\title{
On the role of a conserved, potentially helix-breaking residue in the tRNA-binding $\alpha$-helix of archaeal CCA-adding enzymes
}

\author{
HYUNDAE D. CHO, ${ }^{1,3,4}$ VANITA D. SOOD, ${ }^{1,2,3}$ DAVID BAKER, ${ }^{1,2}$ and ALAN M. WEINER ${ }^{1}$ \\ ${ }^{1}$ Department of Biochemistry, School of Medicine, University of Washington, Seattle, Washington 98195-7350, USA \\ ${ }^{2}$ Howard Hughes Medical Institute, University of Washington, Seattle, Washington 98195-5320, USA
}

\begin{abstract}
Archaeal class I CCA-adding enzymes use a ribonucleoprotein template to build and repair the universally conserved $3^{\prime}$ terminal CCA sequence of the acceptor stem of all tRNAs. A wealth of structural and biochemical data indicate that the Archaeoglobus fulgidus CCA-adding enzyme binds primarily to the tRNA acceptor stem through a long, highly conserved $\alpha$ helix that lies nearly parallel to the acceptor stem and makes many contacts with its sugar-phosphate backbone. Although the geometry of this $\alpha$-helix is nearly ideal in all available cocrystal structures, the helix contains a highly conserved, potentially helix-breaking proline or glycine near the $\mathrm{N}$ terminus. We performed a mutational analysis to dissect the role of this residue in CCA-addition activity. We found that the phylogenetically permissible P295G mutant and the phylogenetically absent P295T had little effect on CCA addition, whereas P295A and P295S progressively interfered with CCA addition (C74>C75 $>$ A76 addition). We also examined the effects of these mutations on tRNA binding and the kinetics of CCA addition, and performed a computational analysis using Rosetta Design to better understand the role of P295 in nucleotide transfer. Our data indicate that CCA-adding activity does not correlate with the stability of the pre-addition cocrystal structures visualized by X-ray crystallography. Rather, the data are consistent with a transient conformational change involving P295 of the tRNA-binding $\alpha$-helix during or between one or more steps in CCA addition.
\end{abstract}

Keywords: CCA addition; minihelix; nucleotidyltransferase; Rosetta Design; tRNA

\section{INTRODUCTION}

The CCA-adding enzyme (ATP[CTP]:tRNA nucleotidyltransferase) is an unusual RNA polymerase that adds a defined sequence of three nucleotides to tRNA without benefit of a nucleic acid template. Although CCA-adding activity is universal except in the minimal genomes of the eubacterial endosymbionts Mycoplasma and Ureaplasma (Mushegian and Koonin 1996), there are two evolutionarily distinct classes of CCA-adding enzymes. Class I enzymes are found in Archaea, class II in eubacteria and eukaryotes (Yue et al. 1996); the two classes of enzymes exhibit no homology outside of the catalytic nucleotidyltransferase

\footnotetext{
${ }^{3}$ These authors contributed equally to this work.

${ }^{4}$ Present address: InVivoScribe Technologies, LLC 6330 Nancy Ridge Drive, Suite 106, San Diego, CA 92121-3230, USA.

Reprint requests to: Alan $M$. Weiner, Department of Biochemistry, School of Medicine, University of Washington, Box 357350, Seattle, WA 98195-7350, USA; e-mail: amweiner@u.washington.edu; fax (206) 685-9231.

Article published online ahead of print. Article and publication date are at http://www.rnajournal.org/cgi/doi/10.1261/rna.1060308.
}

domain that is shared with many RNA and DNA polymerases, aminoglycoside resistance enzymes, and eubacterial regulatory proteins (Aravind and Koonin 1999). CCA-adding enzymes of both classes accept all three incomplete tRNA species as substrates (tRNA-D, tRNADC, and tRNA-DCC, where D is the discriminator base) and faithfully add one nucleotide at a time, using CTP and ATP as precursors to generate mature tRNA. The ability to add CCA without a nucleic acid template is all the more remarkable because the enzyme has only a single active site (Yue et al. 1998) and the tRNA does not translocate along the enzyme as each successive nucleotide is added; thus, the growing $3^{\prime}$ end of the tRNA must refold after each nucleotide addition in order to reposition the new primer terminus for attack on the next incoming NTP within the active site (Shi et al. 1998; Cho et al. 2006).

Complexes of class I CCA-adding enzymes with incomplete tRNAs, mature tRNA, and tRNA substrate mimics have been extensively characterized both biochemically and by X-ray crystallography. All of the crystal structures of the Archaeoglobus fulgidus enzyme, including the apoenzyme 
and the enzyme bound to tRNA or tRNA mimics, are remarkably similar (overall C $\alpha$ RMSD 0.89-2.1 $\AA$ ) with most of the structural variation occurring within the catalytic ("head") domain. The precise mechanism by which the CCA-adding enzyme recognizes and holds the top half of tRNA in the proper register for CCA addition has been subject to some controversy (Xiong and Steitz 2004; Cho et al. 2005; Tomita et al. 2006), but the cocrystal structures agree quite well regarding interactions of the protein with the $3^{\prime}$ end of the acceptor stem and the growing $3^{\prime}$ end of the tRNA substrate. As predicted by the "collaborative templating" model (Shi et al. 1998), the CCA-adding enzyme directs and accommodates refolding of the growing $3^{\prime}$ end of the tRNA; a flexible, highly conserved $\beta$-turn in the head domain plays a key role in this collaborative process, adopting at least three consecutive conformations as the $3^{\prime}$ terminal CCA sequence is added (Shi et al. 1998; Xiong and Steitz 2004; Cho et al. 2006). The detailed mechanism of nucleotide addition has also been revealed at atomic resolution by cocrystallization of the enzyme with and without NTPs and with different tRNA substrates (Xiong and Steitz 2004; Tomita et al. 2006). Nonetheless, despite the wealth of structural and biochemical data, consensus has not been reached regarding the exact mechanism of nucleotide discrimination (Xiong and Steitz 2004; Tomita et al. 2006), the mechanism of the specificity switch from CTP to ATP (Martin et al. 2008), or the role of a progressive conformational change in the head domain relative to the neck, body, and tail (Xiong and Steitz 2004). Finally, and most importantly for this report, X-ray crystallography can only characterize stable structures; transient conformational changes between or even during nucleotide addition steps cannot be visualized and must be inferred from biochemical experiments or from cocrystals with transition state mimics.

In all available cocrystal structures of tRNA or tRNA-like substrates with the class I A. fulgidus CCA-adding enzyme, the acceptor stem of the tRNA substrate is bound to a nearly parallel $\alpha$-helix in the protein (Xiong and Steitz 2004; see Fig. 1A in Tomita et al. 2006). Highly conserved residues near the $\mathrm{N}$ terminus of this $\alpha$-helix bind the tRNA substrate in the proper register for CCA addition; less highly conserved basic residues toward the $\mathrm{C}$ terminus of the $\alpha$-helix contribute to overall binding affinity, but do not appear to affect register (see Fig. 5 in Cho et al. 2006). We had previously noted that residue 295 near the $\mathrm{N}$ terminus of the tRNA-binding $\alpha$-helix is either proline or (less frequently) glycine in 19 of 20 sequenced Archaeal genomes, and we therefore speculated that the $\alpha$-helix might break or partially dislocate at some step in CCA addition (Cho et al. 2006). Genomes encoding several new archaeal CCA-adding enzymes have since been sequenced, and residue 295 is proline or glycine in 32 of 33 homologous archaeal CCA-adding enzymes covering the entire spectrum of known Archaea (Supplemental Fig. 1). We were intrigued that P295 does not cause the tRNA-binding $\alpha$-helix to bend or kink in any of the cocrystal structures of the A. fulgidus enzyme with various tRNA substrates or mimics (Xiong and Steitz 2004; Tomita et al. 2006). On the one hand, proline and glycine are frequently considered helix-breakers (Chakrabartty et al. 1994), and proline can redirect a transmembrane $\alpha$-helix by as much as $30^{\circ}$ (Chakrabarti and Chakrabarti 1998). On the other hand, proline can be readily accommodated within an $\alpha$-helix, where it forms $\mathrm{C}-\mathrm{H} \cdots \mathrm{O}$ hydrogen bonds through the $\delta$ carbon (Chakrabarti and Chakrabarti 1998). In an attempt to reconcile phylogenetic conservation of a potential helixbreaker at residue 295 with structural evidence for a continuous tRNA-binding $\alpha$-helix (Fig. 1), we performed a

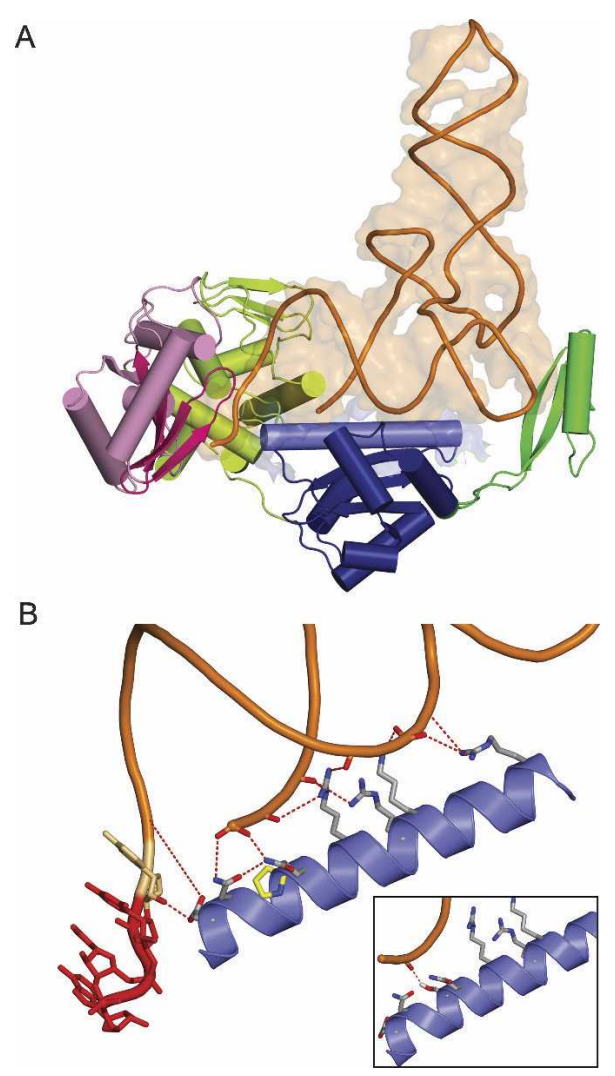

FIGURE 1. The tRNA acceptor stem binds primarily to a tRNAbinding $\alpha$-helix in class I CCA-adding enzymes. (A) Structure of mature tRNA bound to the A. fulgidus CCA-adding enzyme (Xiong and Steitz 2004). tRNA is shown in orange, the head domain of the enzyme in lavender, the neck in light green, the body in blue, and the tail in green. The $\beta$-sheet containing the two catalytic aspartates of the nucleotidyltransferase motif is highlighted in magenta, and the tRNAbinding $\alpha$-helix is shown in lighter blue. $(B)$ Hydrogen bonds between tRNA backbone and side chains extending from the tRNA-binding $\alpha$ helix. Proline 295 is shown in yellow; other side chains are in standard stick representation with carbon gray, nitrogen blue, and oxygen red. See Cho et al. (2006) for residue numbers and a detailed description of the binding interactions. (Inset) Interaction of the tRNA acceptor stem with the tRNA-binding $\alpha$-helix in the P295S mutant. The additional hydrogen bond is indicated by a dashed line. Prepared using PyMol (DeLano 2002). 
mutational analysis designed to determine whether this $\alpha$-helix may undergo a conformational change at P295 (or the equivalent glycine in homologous archaeal enzymes) during one or more steps in CCA addition.

\section{RESULTS AND DISCUSSION}

\section{The effect of mutagenesis of P295 on CCA-addition activity}

To guide our experimental mutational analysis, we first subjected residue 295 in the tRNA-binding $\alpha$-helix to virtual mutagenesis using Rosetta Design (Kuhlman et al. 2003; Ashworth et al. 2006). We replaced P295 with each of the other 19 amino acids and scored the point mutants using a potential energy function that approximates the free energy of the RNP by summing the score of each residue, the individual residue scores themselves being a weighted sum of component scores. (Table 1; data not shown). Only those mutants with a negative (favorable) residue score are shown (Table 1 , column 5 , residue score). The results are sorted by the score of residue 295; the overall score of the RNP complex is also given (Table 1, column 6, RNP score) along with selected components of the residue score. As inferred from the negative residue scores, only five amino acids are structurally compatible with the crystal structure conformation of the tRNAenzyme complex: Pro, Ala, Ser, Gly, and Thr. We therefore expressed and purified histidine-tagged A. fulgidus CCAadding enzymes with each of these P295 mutations and assayed their effect on enzyme activity. As shown in Figure 2, the P295G and P295T mutants displayed close to wildtype activity for all three steps of CCA addition, whereas

TABLE 1. Virtual mutagenesis of residue 295

\begin{tabular}{lrrrrr}
\hline Mutation & LJ-atr & AA-type & H-bond & $\begin{array}{c}\text { Residue } \\
\text { score }\end{array}$ & $\begin{array}{c}\text { RNP } \\
\text { score }\end{array}$ \\
\hline P295 (WT) & -4.3 & 0.1 & -1.3 & -4.8 & -644.70 \\
P295A & -2.7 & -0.2 & -1.3 & -4.3 & -643.49 \\
P295S & -3.2 & 0.2 & -1.8 & -3.7 & -643.30 \\
P295G & -2.1 & 0.4 & -1.3 & -3.2 & -642.07 \\
P295T & -3.5 & 0.2 & -1.3 & -1.9 & -639.66 \\
\hline
\end{tabular}

Rosetta scores for virtual mutagenesis of residue 295. LJ-atr, Lennard-Jones score, attractive portion; AA-type, statistically derived term for the stability of the residue in this $\alpha$-helical context; $\mathrm{H}$-Bond, hydrogen-bonding score; Residue score, total score for residue 295, a weighted sum of component scores given in columns 2-4 and others not shown; RNP score, total score of tRNA-enzyme complex for each mutant enzyme, the weighted sum of all individual residue scores. Note that the Rosetta scores are given in arbitrary units; scores have not been parameterized on the "real" $\Delta \mathrm{G}$ of folding. Several other amino acids were also predicted to favor an $\alpha$-helical conformation of the tRNA-binding helix in the apoenzyme, but both Rosetta calculations and visual inspection indicated that van der Waals clashes with the tRNA would prevent these mutants from binding tRNA substrates.
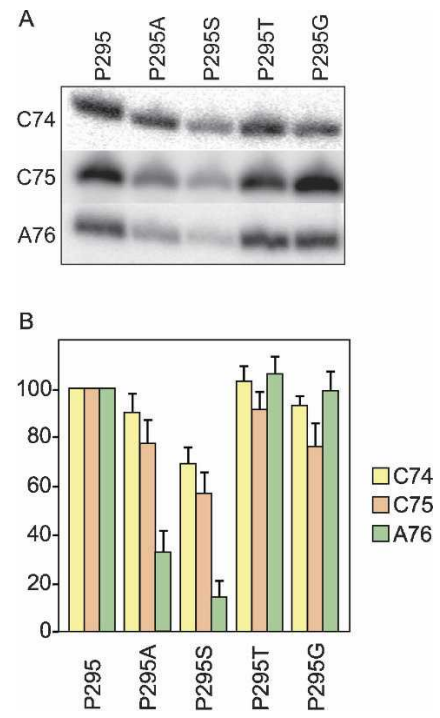

FIGURE 2. Mutations in helix $\mathrm{L}$ of the A. fulgidus CCA-adding enzyme differentially affect addition of C74, C75, or A76. (A) Gel electrophoresis assay of the enzymes with tRNA substrates lacking A, CA, or CCA (tRNA-NCC, tRNA-NC, or tRNA-N, where $\mathrm{N}$ is the discriminator base), all four unlabeled nucleotides, and $\left[\alpha-{ }^{32} \mathrm{P}\right]-$ labeled CTP or ATP as appropriate. Standard assays in the linear range for wild-type enzyme. $(B)$ Activity of enzymes relative to wild type (P295). The average of three independent experiments is shown, with error bars indicating standard deviation.

the P295A and P295S mutants were less active than wild type and progressively more impaired for each successive nucleotide addition (C74>C75 $>>$ A76). Although it was unsurprising that the phylogenetically permissible P295G mutant would retain wild-type activity, the high activity of the phylogenetically absent P295T mutant and the impaired activity of the structurally compatible P295S and P295A mutants was puzzling, and we therefore attempted to correlate the individual components of the Rosetta Design scores with the activities of the mutant enzymes.

We first considered the P295A mutant. In principle, the impaired activity of this mutant compared with the wildtype P295 could reflect loss of interdomain van der Waals interactions: Note the lower (more favorable) score of P295 compared with $\mathrm{P} 295 \mathrm{~A}$ in the Lennard-Jones attractive (LJ-atr) portion of the Rosetta scores (Table 1, column 2). However, P295G is also destabilized in the LJ-atr component of the Rosetta score (Table 1), yet it displays wild-type activity (Fig. 2). This indicates that loss of interdomain van der Waals interactions cannot account for the decreased activity of the P295A mutant. As we had previously hypothesized that P295 might be involved in a transient conformational change, we examined the effect of mutations at position 295 on the predicted stability of the tRNAbinding $\alpha$-helix. In fact, P295A is the only mutation that stabilizes the helical backbone conformation at position 295 (Table 1, column 3, AA-type), consistent with the known property of alanine as a strong helix former 
(Chakrabartty et al. 1994). Taken together with the destabilization of the helical conformation in the highly active P295G and P295T mutants (Table 1, column 3), the effects of these mutations suggest that CCA-adding activity correlates with helix destabilization at residue 295.

Rosetta Design predicts that the P295S mutant, like P295G or P295T, destabilizes the helical backbone conformation of the tRNA-binding $\alpha$-helix (Table 1, column 3), yet P295S decreases enzyme activity (Fig. 2). This seemingly contradictory result is not difficult to explain, because Rosetta Design also predicts that the $\gamma$-hydroxyl of serine 295 makes an additional hydrogen bond between the tRNA-binding $\alpha$-helix and the tRNA acceptor stem (Fig. $1 \mathrm{~B}$, inset). This would stabilize the tRNA-binding $\alpha$-helix in the P295S mutant (Table 1, column 4, H-bond), consistent with our hypothesis that enzyme activity correlates with destabilization of the structure in the vicinity of residue 295.

\section{Mutations at P295 do not affect tRNA binding}

The progressive inhibition of $\mathrm{C} 74>\mathrm{C} 75>>\mathrm{A} 76$ addition seen for P295A and P296S (Fig. 2) could be caused by defects in tRNA binding, NTP binding, catalysis, or some intermediate reaction step. To separate these effects, we compared binding of tRNA-N, tRNA-NC, and tRNA-NCC to the P295, P295A, and P296S enzymes by electrophoretic mobility shift assays (summarized in Table 2). We considered it unlikely that stabilization of the tRNA-binding $\alpha$-helix by the P295A mutation, or creation of an extra hydrogen bond to the tRNA by the P295S mutation, would significantly weaken formation of the tRNA-enzyme complex, and, indeed, the $K_{\mathrm{d}}$ for enzyme binding to each tRNA processing intermediate was affected by 4.5 -fold or less (Table 2). This is consistent with the Rosetta prediction that the P295A and P295S mutations would have little effect on the stability of the cocrystal structures (Table 1, columns 5,6), and also with our previous evidence that tRNA binding is almost entirely dependent on interactions with the acceptor stem and is not significantly affected by binding of the growing 3 -terminal CCA sequence within the active site (Cho et al. 2006). Thus, the significant defects in CCA-adding activity displayed by these mutants (Fig. 1) are likely to reflect impairment of one or more steps in CCA addition subsequent to initial tRNA binding.

TABLE 2. Dissociation constants $\left(K_{\mathrm{d}}, \mu \mathrm{M}\right)$ for binding of tRNA substrates to the CCA-adding enzyme calculated from electrophoretic mobility shift assays

\begin{tabular}{lccc}
\hline & tRNA-N & tRNA-NC & tRNA-NCC \\
\hline P295 (WT) & 0.08 & 0.13 & 0.14 \\
P295A & 0.17 & 0.47 & 0.15 \\
P295S & 0.18 & 0.55 & 0.24 \\
\hline
\end{tabular}

\section{Defects in CCA-addition activity correlate with increases in apparent $K_{\mathrm{m}}$ for NTPs and decreases in catalytic efficiency}

As the identity of residue 295 in the tRNA-binding $\alpha$-helix does not appear to affect tRNA binding (Table 2), a subsequent step in the complex CCA-addition reaction must be responsible for the effects of mutating P295. We therefore determined an apparent $K_{\mathrm{m}}$ and $k_{\text {cat }}$ for CTP and ATP using the natural substrates tRNA-N, tRNA-NC, and tRNA-NCC (Table 3). As expected, the P295T and P295G mutants have little effect on $K_{\mathrm{m}}$ and $k_{\text {cat }}$ for C74, C75, and A76 addition. P295A has relatively small effects on C74 and C75 addition; however, the $K_{\mathrm{m}}$ for ATP increases by about 26 -fold and $k_{\text {cat }}$ decreases by about threefold, reducing overall catalytic efficiency $\left(k_{\mathrm{cat}} / K_{\mathrm{m}}\right)$ by two orders of magnitude. P295S progressively increased $K_{\mathrm{m}}$ for each NTP addition by 20 - to 40 -fold, while decreasing $k_{\text {cat }}$ by no more than threefold; this reduced catalytic efficiency for each nucleotide addition step by two orders of magnitude or more (Table 3 ).

In the original Michaelis-Menten equation of enzyme kinetics (Michaelis and Menten 1913), $k_{3}$, which for our purposes represents the observed rate constant for all steps subsequent to NTP binding to the binary tRNA-enzyme complex, was assumed to be much smaller than the off-rate for NTP binding. As a result, the Michaelis-Menten constant $\left(K_{\mathrm{m}}\right)$ for NTP addition was equivalent to the equilibrium dissociation constant $\left(K_{\mathrm{d}}\right)$, or affinity, of the binary complex for the NTP. Briggs and Haldane (1925) showed that this assumption was unnecessary, and that $K_{\mathrm{m}}$ could more correctly be defined as an upper limit for the $K_{\mathrm{d}}$ of the NTP with the binary tRNA-enzyme complex under steadystate conditions. However, even this generalization fails to hold for more complex reactions; the Michaelis-Menten constant may even be smaller than the dissociation constant, depending on the complexity of the enzyme reaction pathway and the relative magnitude of each elemental rate constant (Northrop 1998). For a complex reaction such as CCA-addition, where little is known about the elemental rate constants, and at least six molecular species (tRNA-N, tRNA-NC, tRNA-NCC, tRNA-CCA, CTP, and ATP) compete for binding to overlapping sites, the meaning of the Michaelis-Menten constant cannot be precisely defined as referring to binding of a particular substrate; nor can catalytic efficiency $\left(k_{\text {cat }} / K_{\mathrm{m}}\right)$ be associated with a particular step such as catalysis or polymerization. Rather, these terms must be interpreted as referring to any, and perhaps all steps from NTP binding to catalysis to product release.

The CCA-adding enzymes may be partially processive, further complicating the use of Michaelis-Menten kinetics. On the one hand, the ability of the enzymes to use all three incomplete tRNAs as substrate (tRNA-N, tRNA-NC, and tRNA-NCC), as well as the existence of organisms where CCA-addition is the joint responsibility of CC-adding and 


\section{Cho et al.}

TABLE 3. Steady-state kinetic parameters for P295 mutants

\begin{tabular}{|c|c|c|c|c|c|c|c|c|c|}
\hline \multirow[b]{2}{*}{ Enzyme } & \multicolumn{3}{|c|}{ C74 addition } & \multicolumn{3}{|c|}{ C75 addition } & \multicolumn{3}{|c|}{ A76 addition } \\
\hline & $K_{\mathrm{m}}$ & $k_{\text {cat }}$ & $k_{\text {cat }} / K_{\mathrm{m}}(\mathrm{rel})$ & $K_{\mathrm{m}}$ & $k_{\text {cat }}$ & $k_{\mathrm{cat}} / K_{\mathrm{m}}(\mathrm{rel})$ & $K_{\mathrm{m}}$ & $k_{\text {cat }}$ & $k_{\mathrm{cat}} / K_{\mathrm{m}}(\mathrm{rel})$ \\
\hline WT & 10 & 3.68 & 1 & 17.5 & 3.21 & 1 & 300 & 2.56 & 1 \\
\hline A295 & 37 & 3.53 & 0.28 & 40 & 2.49 & 0.33 & 7640 & 0.83 & 0.01 \\
\hline S295 & 200 & 2.55 & 0.03 & 650 & 1.83 & 0.01 & 12,500 & 0.76 & 0.01 \\
\hline T295 & 25 & 3.80 & 0.36 & 25 & 2.93 & 0.67 & 350 & 2.72 & 0.92 \\
\hline G295 & 10 & 3.43 & 0.94 & 90 & 2.46 & 0.15 & 233 & 2.55 & 1.29 \\
\hline
\end{tabular}

A-adding enzymes (Tomita and Weiner 2001, 2002), argue against strict processivity. On the other hand, the presence in both Archaea and Gram-positive Eubacteria of tRNA genes encoding either all or none of CCA, but never part of it, is most easily rationalized by processivity: There would be no selection for encoded $\mathrm{C} 74$ or $\mathrm{C} 75$, because a processive enzyme would quickly add these two nucleotides once tRNA-N was bound.

In conclusion, the P295A and P295S mutants have large effects on the $K_{\mathrm{m}}$ for NTP addition, small effects on tRNA binding, and are spatially distant from the nucleotide binding site. This suggests that residue 295 is involved in some step(s) other than substrate binding or catalysis. Moreover, the greater the calculated stability of the tRNA-binding $\alpha$-helix as seen in various cocrystal structures, the lower the CCA-adding activity with $\mathrm{C} 74>\mathrm{C} 75>\mathrm{A} 76$ addition. This suggests that P295 undergoes a transient conformational change during or between one or more steps of CCA addition, and that this conformational change becomes progressively more important or difficult as each new nucleotide is added. The proposed conformational change would explain the conservation of a "helix-breaking" residue at position 295 of the tRNA-binding $\alpha$-helix in archaeal CCA-adding enzymes, and would highlight how much we still have to learn about the mechanism of this unique RNA polymerase.

\section{MATERIALS AND METHODS}

\section{Virtual mutagenesis}

Using the Rosetta Design program (Kuhlman et al. 2003; Ashworth et al. 2006), all 20 amino acids were substituted at position 295 of $A$. fulgidus CCA-adding enzyme (PDB code 1SZ1) (Xiong and Steitz 2004), while allowing the conformations of nearby contacting residues to vary. Side-chain conformations were from the Dunbrack backbone-dependent rotamer library (Bower et al. 1997), supplemented with the native side-chain conformations from the crystal structure. A Monte Carlo search procedure was used to identify the combination of rotamers with the lowest energy according to a potential that includes a Lennard-Jones potential to describe atomic packing interactions (van der Waals forces), an orientation-dependent hydrogen-bonding potential
(Kortemme et al. 2003), statistical terms approximating the backbone-dependent amino acid type and rotamer probabilities (Kuhlman et al. 2003), a generalized Born model of electrostatics (Ashworth et al. 2006), an implicit solvation model (Lazaridis and Karplus 1999), and an estimate of unfolded reference state energies (Kuhlman et al. 2003). Only protein-protein and protein-RNA interactions were considered in the final score; intramolecular RNA interactions were constant in each virtual point mutant and were not used, because the RNA energy would simply result in a constant offset to the final score. The relative stability of the enzyme-tRNA complex in the context of each amino acid substitution was scored, as well as the stability of mutated residue itself.

We were initially concerned that the relatively low resolution of the cocrystal structures (Xiong and Steitz 2004) might affect the Rosetta calculations; however, what ultimately matters is not the resolution of the diffraction data, but how good a model is made from those data, and, in fact, we obtained essentially identical results (data not shown) using the higher resolution data of Tomita et al. (2006). We therefore chose to present our results using the more biologically relevant cocrystal structure of the enzyme complexed with a natural tRNA substrate. There are several reasons why the signal-to-noise ratio is much less worrisome for this unusual application of Rosetta than it would be for more typical uses of a protein design program. Even at lower resolution, the backbone of the protein is almost certainly correct, and those are the only coordinates necessary for accurate AA-type scores, which is one key component in our calculations. The other key component of our calculations is the hydrogen bonding score, and this is easily verified as a real computational signal by visual inspection of the modeled hydrogen bond. In addition, one would not expect a larger signal for "real" hydrogen bonds than for incorrect ones. If the design program makes a mistake, it assigns just as good a score to the mistake as to a correctly predicted bond. Thus, although the various virtual mutants exhibit very similar Rosetta scores, this is the expected result for mutating a single residue and repacking only a few nearby residues, no matter how high the resolution of the data used to build starting model. If many mutations were being made at once, small inaccuracies in the score function could add up, and one would indeed expect to see larger differences in the RNP scores.

\section{Mutagenesis and enzyme assays}

Mutations were introduced into the A. fulgidus CCAadding enzyme using the QuickChange (Stratagene) kit, and 
hexahistidine-tagged enzymes were prepared as described (Cho et al. 2003). tRNA substrates lacking A, CA, or CCA were prepared as described (Cho et al. 2002, 2003) by runoff transcription from a Bacillus subtilis aspartate tRNA template (Oh and Pace 1994) or modifications thereof (Cho et al. 2003). Standard $10 \mu \mathrm{L}$ CCA addition assays were performed as described (Cho et al. 2003).

Electrophoretic mobility shift assays were performed as described (Shi et al. 1998) and quantified by Storm PhosphorImager (Amersham Biosciences). The ratio of free to bound tRNA was measured as a function of enzyme concentration, and $K_{\mathrm{d}}$ calculated from the slope of a double reciprocal plot (Carey and Uhlenbeck 1983).

Kinetic parameters were assayed by addition of $\left[\alpha-{ }^{32} \mathrm{P}\right] \mathrm{CTP}$ to tRNA-N or tRNA-NC and $\left[\alpha-{ }^{32} \mathrm{P}\right]$ ATP to tRNA-NCC under standard assay conditions in the presence of all four unlabeled nucleotides (100 $\mu \mathrm{M}$ each), $40 \mathrm{nM}$ enzyme, and $2 \mu \mathrm{M}$ tRNA-N, tRNA-NC, or tRNA-NCC ( $>3.5$-fold excess over the $K_{d}$ for even the weakest binding mutant; see Table 2). For analysis of C74 addition, electrophoresis was performed for a short enough time to allow comigration of $\mathrm{C} 74$ and $\mathrm{C} 75$ addition products; these were quantified together, as any $\mathrm{C} 75$ addition product must by definition include a C74 addition. Linear-reciprocal plots of initial velocities against NTP concentrations were used to determine $K_{\mathrm{m}}$ values for NTP addition. CTP concentrations were varied from 6.25 to $50 \mu \mathrm{M}$ for the $\mathrm{C} 74$ and $\mathrm{C} 75$ addition reactions, and ATP concentrations from 250 to $2000 \mu \mathrm{M}$ for the A76 reaction. Incorporation was measured in arbitrary Storm PhosphorImager (Amersham Biosciences) units proportional to counts per minute.

\section{SUPPLEMENTAL DATA}

Supplemental material can be found at http://www.rnajournal.org.

\section{ACKNOWLEDGMENTS}

We thank Arnold Bailey and Tom Pavelitz for advice. V.D.S. was supported by the Canadian Institutes for Health Research. This work was supported by National Institutes of Health Grants GM059804 (A.M.W.) and GM059224 (D.B.), and the Howard Hughes Medical Institute (D.B.).

Received March 4, 2008; accepted April 18, 2008.

\section{REFERENCES}

Aravind, L. and Koonin, E.V. 1999. DNA polymerase $\beta$-like nucleotidyltransferase superfamily: Identification of three new families, classification and evolutionary history. Nucleic Acids Res. 27: $1609-1618$.

Ashworth, J., Havranek, J.J., Duarte, C.M., Sussman, D., Monnat Jr., R.J., Stoddard, B.L., and Baker, D. 2006. Computational redesign of endonuclease DNA binding and cleavage specificity. Nature 441: 656-659.

Bower, M.J., Cohen, F.E., and Dunbrack Jr., R.L. 1997. Prediction of protein side-chain rotamers from a backbone-dependent rotamer library: A new homology modeling tool. J. Mol. Biol. 267: 12681282.

Briggs, G.E. and Haldane, J.B. 1925. A note on the kinetics of enzyme action. Biochem. J. 19: 338-339.

Carey, J. and Uhlenbeck, O.C. 1983. Kinetic and thermodynamic characterization of the R17 coat protein-ribonucleic acid interaction. Biochemistry 22: 2610-2615.
Chakrabarti, P. and Chakrabarti, S. 1998. C-H $\cdots$ O hydrogen bond involving proline residues in $\alpha$-helices. J. Mol. Biol. 284: 867-873.

Chakrabartty, A., Kortemme, T., and Baldwin, R.L. 1994. Helix propensities of the amino acids measured in alanine-based peptides without helix-stabilizing side-chain interactions. Protein Sci. 3: 843-852.

Cho, H.D., Tomita, K., Suzuki, T., and Weiner, A.M. 2002. U2 small nuclear RNA is a substrate for the CCA-adding enzyme (tRNA nucleotidyltransferase). J. Biol. Chem. 277: 3447-3455.

Cho, H.D., Oyelere, A.K., Strobel, S.A., and Weiner, A.M. 2003. Use of nucleotide analogs by class I and class II CCA-adding enzymes (tRNA nucleotidyltransferase): Deciphering the basis for nucleotide selection. RNA 9: 970-981.

Cho, H.D., Verlinde, C.L., and Weiner, A.M. 2005. Archaeal CCAadding enzymes: Central role of a highly conserved $\beta$-turn motif in RNA polymerization without translocation. J. Biol. Chem. 280: 9555-9566.

Cho, H.D., Chen, Y., Varani, G., and Weiner, A.M. 2006. A model for C74 addition by CCA-adding enzymes: C74 addition, like C75 and A76 addition, does not involve tRNA translocation. J. Biol. Chem. 281: 9801-9811.

DeLano, W.L. 2002. The pymol molecular graphics system. DeLano Scientific, San Carlos, CA. http://www.pymol.org.

Kortemme, T., Morozov, A.V., and Baker, D. 2003. An orientationdependent hydrogen bonding potential improves prediction of specificity and structure for proteins and protein-protein complexes. J. Mol. Biol. 326: 1239-1259.

Kuhlman, B., Dantas, G., Ireton, G.C., Varani, G., Stoddard, B.L., and Baker, D. 2003. Design of a novel globular protein fold with atomic-level accuracy. Science 302: 1364-1368.

Lazaridis, T. and Karplus, M. 1999. Effective energy function for proteins in solution. Proteins 35: 133-152.

Martin, G., Doublie, S., and Keller, W. 2008. Determinants of substrate specificity in RNA-dependent nucleotidyltransferases. Biochim. Biophys. Acta 1779: 206-216.

Michaelis, L. and Menten, M. 1913. Die Kinetik der Invertinwirkung. Biochem. Z. 49: 333-369.

Mushegian, A.R. and Koonin, E.V. 1996. A minimal gene set for cellular life derived by comparison of complete bacterial genomes. Proc. Natl. Acad. Sci. 93: 10268-10273.

Northrop, D.B. 1998. On the meaning of $K_{\mathrm{m}}$ and $V / K$ in enzyme kinetics. J. Chem. Educ. 75: 1153-1157.

Oh, B.K. and Pace, N.R. 1994. Interaction of the 3 '-end of tRNA with ribonuclease P RNA. Nucleic Acids Res. 22: 4087-4094.

Shi, P.-Y., Weiner, A.M., and Maizels, N. 1998. A top-half tDNA minihelix is a good substrate for the eubacterial CCA-adding enzyme. RNA 4: 276-284.

Tomita, K. and Weiner, A.M. 2001. Collaboration between CC- and A-adding enzymes to build and repair the $3^{\prime}$-terminal CCA of tRNA in Aquifex aeolicus. Science 294: 1334-1336.

Tomita, K. and Weiner, A.M. 2002. Closely related CC- and A-adding enzymes collaborate to construct and repair the $3^{\prime}$-terminal CCA of tRNA in Synechocystis sp. and Deinococcus radiodurans. J. Biol. Chem. 277: 48192-48198.

Tomita, K., Ishitani, R., Fukai, S., and Nureki, O. 2006. Complete crystallographic analysis of the dynamics of CCA sequence addition. Nature 443: 956-960.

Xiong, Y. and Steitz, T.A. 2004. Mechanism of transfer RNA maturation by CCA-adding enzyme without using an oligonucleotide template. Nature 430: 640-645.

Yue, D., Maizels, N., and Weiner, A.M. 1996. CCA-adding enzymes and poly(A) polymerases are all members of the same nucleotidyltransferase superfamily: Characterization of the CCA-adding enzyme from the archaeal hyperthermophile Sulfolobus shibatae. RNA 2: 895-908.

Yue, D., Weiner, A.M., and Maizels, N. 1998. The CCAadding enzyme has a single active site. J. Biol. Chem. 273: 29693-29700. 

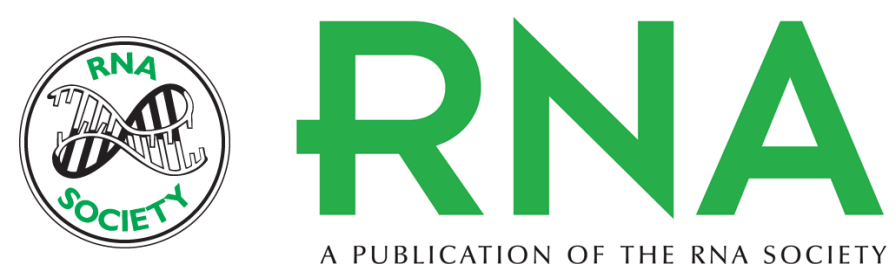

A PUBLICATION OF THE RNA SOCIETY

\section{On the role of a conserved, potentially helix-breaking residue in the tRNA-binding $\alpha$-helix of archaeal CCA-adding enzymes}

Hyundae D. Cho, Vanita D. Sood, David Baker, et al.

RNA 2008 14: 1284-1289

Supplemental Material

References

License

Email Alerting Service
http://rnajournal.cshlp.org/content/suppl/2008/05/22/rna.1060308.DC1

This article cites 26 articles, 12 of which can be accessed free at: http://rnajournal.cshlp.org/content/14/7/1284.full.html\#ref-list-1

Receive free email alerts when new articles cite this article - sign up in the box at the top right corner of the article or click here. 